\title{
Exact and Accurate Reanalysis of Structures for Geometrical Changes
}

\author{
U. Kirsch ${ }^{1}$ and P. Y. Papalambros ${ }^{2}$ \\ ${ }^{1}$ Department of Civil Engineering Technion, Israel Institute of Technology, Haifa, Israel; ${ }^{2}$ Department of Mechanical Engineering, \\ University of Michigan, Ann Arbor, MI, USA
}

\begin{abstract}
A reanalysis approach for geometrical changes in structural systems is presented. The solution procedure is based on the combined approximations method, where the binomial series terms are used as basis vectors in reduced basis approximations. The calculations are based on results of a single exact analysis, calculation of derivatives is not required, and each reanalysis involves a small computational effort. The method is easy to implement, and can be used with general finite element programs. Exact solutions are obtained efficiently for low-rank modifications in the geometry. Accurate solutions are achieved in cases where the basis vectors come close to being linearly dependent. Such solutions are also achieved for nearly scaled geometries, when the angle between the two vectors representing the initial design and modified design is small. Numerical examples demonstrate the high accuracy achieved with a small number of basis vectors.
\end{abstract}

Keywords. Approximate reanalysis; Geometrical optimization; Structural optimization

\section{Introduction}

The structural response cannot usually be expressed explicitly in terms of the structure properties, and structural analysis involves the solution of a set of simultaneous equations. Multiple repeated analyses are needed in various structural analysis, design and optimisation problems. In a typical design process, it is necessary to analyse repeatedly modified structures due to changes in the design. In structural optimisation problems, the cross-sections of elements, the geometry of the structure (coordinates of joints) and the topology (number and orientation of elements and joints) are changed successively

Correspondence and offprint requests to: Professor U. Kirsch, Department of Civil Engineering, Technion, Israel Institute of Technology, Technion City, Haifa, 32000, Israel. Email: kirsch@tx.technion.ac.il until the optimum is reached. In many design and optimisation tasks the analysis part will require most of the computational effort. The high computational cost involved in repeated analysis of large-scale problems is one of the main obstacles involved in the optimisation of such problems. Therefore, only methods that do not involve numerous time consuming implicit analyses might be considered.

Reanalysis procedures are intended to analyse efficiently modified structures due to changes in the structure properties. The goal is to evaluate the structural response (e.g. displacements, stresses and forces) for such changes without solving the complete set of modified implicit equations. Reanalysis methods can be divided broadly into exact (directclosed form) and approximate methods. In this study, both exact and approximate solutions will be considered.

Several exact methods for calculating the modified response due to changes in the design have been proposed in the past. Most of these methods are based on the Sherman-Morrison-Woodbury formulae $[1,2]$. Exact methods are suitable for changes in a relatively small number of elements, and are inefficient in cases of changes in a large proportion of the structure. Improved versions of the ShermanMorrison-Woodbury approach have been proposed by several authors $[3,4]$.

Approximate methods are more efficient than exact methods, and are usually suitable for moderate changes in the whole structure. In general, the efficiency of the calculations and quality of the results are conflicting factors that should be considered. Better approximations are often achieved at the expense of more computational effort. Approximate methods can be divided into the following classes [5,6]:

(a) Global (multi-point) approximations, such as reduced basis [7] and response surface methods 
[8]. These approximations are obtained by analyzing the structure at a number of design points, and they are valid for the whole design space. However, global approximations may require much computational effort, particularly in problems with large number of design variables.

(b) Local (single-point) approximations, such as the first-order Taylor series expansion or the binomial series expansion about a given design point. Local approximations are based on information calculated at a single point. These methods are more efficient, but they are effective only in cases of small changes in the design variables. To improve the quality of the results, various means have been proposed $[9,10]$.

In this study a third class, attempting to give global qualities to local approximations, is considered. The method has been used in previous studies for various problems and different types of design variables [11-17]. The solution procedure is based on the Combined Approximations (CA) approach, where the binomial series terms are used as basis vectors in reduced basis approximations. Similar to local approximations, the calculations are based on results of a single exact analysis. Each reanalysis involves a small computational effort, and calculation of derivatives is not required. The method is easy to implement and can be used with general finite elements programs.

The changes in the structure usually describe member cross-section sizes, the topology and the geometry. Cross-sectional changes are the simplest, since the response functions are often nearly linear functions of some cross-sectional variables. Topological changes are more complicated, particularly in cases where the number of degrees of freedom is changed. In previous studies, reanalysis procedures have been developed for topological optimisation [11,15,17]. Geometrical changes are conceptually similar to cross-section changes, in the sense that the number of degrees of freedom is unchanged. However, since the response is highly nonlinear function of the design variables, it is difficult to achieve accurate approximations.

In this paper, both exact and approximate solutions for geometrical changes are presented. Exact solutions are obtained efficiently by the CA method in cases of low-rank modifications in the structure. For changes affecting a large proportion of the structure, approximate solutions are more efficient. Accurate approximations are achieved in many cases where the basis vectors come close to being linearly dependent. Such solutions are also achieved for nearly scaled geometries, when the angle between the two vectors representing the initial design and modified design is small.

In Section 2, the reanalysis problem under consideration is formulated. A procedure to obtain approximate solutions by the CA method is briefly described in Section 3. Exact solutions for low-rank modifications are introduced in Section 4, and some general cases of accurate solutions for high-rank modifications are developed in Section 5. Numerical examples demonstrate the accuracy of the results in Section 6, and the conclusions are drawn in Section 7.

\section{Problem Formulation}

The reanalysis problem considered in this study can be stated as follows:

(a) Given an initial design, the corresponding stiffness matrix $\mathbf{K}_{0}$, and the initial load vector $\mathbf{R}_{0}$. The corresponding displacements $\mathbf{r}_{0}$ are computed by the equilibrium equations

$$
\mathbf{K}_{0} \mathbf{r}_{0}=\mathbf{R}_{0}
$$

It is assumed that the stiffness matrix $\mathbf{K}_{0}$ is given from the initial analysis in the decomposed form:

$$
\mathbf{K}_{0}=\mathbf{U}_{0}^{T} \mathbf{U}_{0}
$$

where $\mathbf{U}_{0}$ is an upper triangular matrix.

(b) Assume changes in the geometry (coordinates of joints) so that the modified stiffness matrix $\mathbf{K}$ and the modified load vector $\mathbf{R}$ are given by

$$
\begin{aligned}
& \mathbf{K}=\mathbf{K}_{0}+\Delta \mathbf{K} \\
& \mathbf{R}=\mathbf{R}_{0}+\Delta \mathbf{R}
\end{aligned}
$$

where $\Delta \mathbf{K}$ and $\Delta \mathbf{R}$ are the changes in the stiffness matrix and in the load vector, respectively, due to change in the geometry.

(c) The goal is to find efficient and accurate approximations of the modified displacements $\mathbf{r}$ due to various changes in the geometry, without solving the complete set of modified analysis equations

$$
\mathbf{K r}=\left(\mathbf{K}_{0}+\Delta \mathbf{K}\right) \mathbf{r}=\mathbf{R}_{0}+\Delta \mathbf{R}
$$

Once the displacements are evaluated, the explicit stress-displacement relations can readily determine the stresses. Thus, the presented approximations of 
$\mathbf{r}$ are intended only to replace the set of implicit analysis Eqs (4).

The above formulation is general, and it is suitable for different types of design variables and structures. For illustrative purposes, discrete structures are considered in this study, but the approach presented is suitable also for shape changes in continuum structures.

\section{Approximate Reanalysis}

In the procedure presented below, the computed terms of the binomial series expansion are used as high quality basis vectors in reduced basis approximations. The unknown coefficients of the reduced basis expression are determined by solving a reduced set of the analysis equations. The efficiency and accuracy are further improved by introducing an uncoupled set of basis vectors, using a GramSchmidt orthonormalization. A detailed discussion of the solution process is given elsewhere [12]. For completeness of presentation, evaluation of the displacements by the CA method is briefly described in this section.

Given the initial stiffness matrix $\mathbf{K}_{0}$ in the decomposed form of Eq. (2), the initial loads $\mathbf{R}_{0}$ and the initial displacements $\mathbf{r}_{0}$, calculation of the modified displacements $\mathbf{r}$ for any assumed changes $\Delta \mathbf{K}, \Delta \mathbf{R}$, in the stiffness matrix and in the load vector, involves the following steps:

(a) The modified stiffness matrix $\mathbf{K}$ and the modified load vector $\mathbf{R}$ are first introduced. Since $\mathbf{K}_{0}$ and $\mathbf{R}_{0}$ are given, this step involves only introduction of $\Delta \mathbf{K}$ and $\Delta \mathbf{R}$

(b) The basis vectors $\mathbf{r}_{i}$ are calculated. Define matrix $\mathbf{B}$ as

$$
\mathbf{B}=\mathbf{K}_{0}^{-1} \Delta \mathbf{K}
$$

Pre-multiplying Eq. (4) by $\mathbf{K}_{0}^{-1}$, substituting Eqs (1) and (5), and premultiplying the resulting equation by $(\mathbf{I}+\mathbf{B})^{-1}$, gives the following expression for the displacements:

$$
\mathbf{r}=(\mathbf{I}+\mathbf{B})^{-1} \mathbf{r}_{0}
$$

For small changes $\Delta \mathbf{K}$ this expression can be approximated by the binomial series

$$
\mathbf{r}=\left(\mathrm{I}-\mathbf{B}+\mathbf{B}^{2}-, \ldots,+\mathbf{B}^{\mathrm{s}-1}\right) \mathbf{r}_{0}
$$

Equation (7) is the series of basis vectors, defined as

$$
\begin{aligned}
\mathbf{r}_{1} & =\mathbf{K}_{0}^{-1} \mathbf{R} \\
\mathbf{r}_{i} & =-\mathbf{B} \mathbf{r}_{i-1} \quad i=2, \ldots, s
\end{aligned}
$$

Where $s$ is the number of vectors considered (it is assumed that $s \ll$ number of degrees of freedom). The matrix of the basis vectors $\mathbf{r}_{B}$ is defined by

$$
\mathbf{r}_{B}=\left[\mathbf{r}_{1}, \mathbf{r}_{2}, \ldots, \mathbf{r}_{s}\right]
$$

In cases where $\Delta \mathbf{R}=\mathbf{0}$, the first basis vector is simply $\mathbf{r}_{1}=\mathbf{r}_{0}$. Calculation of the basis vectors by Eq. (8) involves only forward and backward substitutions in cases where $\mathbf{K}_{0}$ is available in the form of Eq. (2) from the initial analysis of the structure. For example, assuming that $\mathbf{r}_{1}$ is given, the vector $\mathbf{r}_{2}$ is calculated by

$$
\mathbf{K}_{0} r_{2}=-\Delta \mathbf{K} \mathbf{r}_{1}
$$

We solve first for the vector of unknowns $\mathbf{t}$ by the forward substitution

$$
\mathbf{U}_{0}^{T} \mathbf{t}=-\Delta \mathbf{K} \mathbf{r}_{1}
$$

The vector $\mathbf{r}_{2}$ is then calculated by the backward substitution

$$
\mathbf{U}_{0} \mathbf{r}_{2}=\mathbf{t}
$$

Similarly, $\mathbf{r}_{3}$ is calculated by

$$
\mathbf{K}_{0} \mathbf{r}_{3}=-\Delta \mathbf{K} \mathbf{r}_{2}
$$

(c) The reduced matrix $\mathbf{K}_{R}$, and the reduced vector $\mathbf{R}_{R}$, are calculated by

$$
\mathbf{K}_{R}=\mathbf{r}_{B}^{T} \mathbf{K} \mathbf{r}_{B} \quad \mathbf{R}_{R}=\mathbf{r}_{B}^{T} \mathbf{R}
$$

(d) The vector of unknown coefficients $\mathbf{y}$ is calculated by solving the set of $(s \times s)$ equations

$$
\mathbf{K}_{R} \mathbf{y}=\mathbf{R}_{R}
$$

(e) The modified displacements $\mathbf{r}$ are evaluated by

$$
\mathbf{r}=y_{1} \mathbf{r}_{1}+y_{2} \mathbf{r}_{2}+\ldots+y_{s} \mathbf{r}_{s}=\mathbf{r}_{B} \mathbf{y}
$$

To improve the efficiency and accuracy of the approximations, the reduced set of simultaneous Eqs (15) can be transformed into an uncoupled form. An uncoupled set of new basis vectors $\mathbf{V}_{i}$ $(i=1,2, \ldots, s)$ is introduced using a Gram-Schmidt orthonormalisation $[12,18]$. The new vectors are determined by the original ones $\mathbf{r}_{i}$ from

$$
\begin{aligned}
& \mathbf{V}_{1}=\left|\mathbf{r}_{1}^{T} \mathbf{K} \mathbf{r}_{1}\right|^{-1 / 2} \mathbf{r}_{1} \\
& \overline{\mathbf{V}}_{i}=\mathbf{r}_{i}-\sum_{j=1}^{i-1}\left(\mathbf{r}_{i}^{T} \mathbf{K} \mathbf{V}_{j}\right) \mathbf{V}_{j} \\
& \mathbf{V}_{i}=\left|\overline{\mathbf{V}}_{i}^{T} \mathbf{K} \overline{\mathbf{V}}_{i}\right|^{-1 / 2} \overline{\mathbf{V}}_{i} \quad i=2, \ldots, s
\end{aligned}
$$


where $\overline{\mathbf{V}}_{i}$ and $\mathbf{V}_{i}$ are the $i$ th non-normalised and normalised vectors, respectively. Defining the matrix $\mathbf{V}_{B}$ of new basis vectors and the vector $\mathbf{z}$ of new coefficients, the reduced system of Eq. (15) becomes uncoupled, and the final displacements are given by the explicit expression

$$
\mathbf{r}=\mathbf{V}_{B} \mathbf{z}=\mathbf{V}_{B}\left(\mathbf{V}_{B}^{T} \mathbf{R}\right)
$$

The displacements calculated by Eq. (19) can be expressed as an additively separable quadratic function of the basis vectors $\mathbf{V}_{i}$ by

$$
\mathbf{r}=\sum_{i=1}^{s} \mathbf{V}_{i}\left(\mathbf{V}_{i}^{T} \mathbf{R}\right)
$$

For any assumed number of basis vectors, identical results are obtained by considering either the original set of basis vectors or the new set of uncoupled basis vectors. The advantage in using the new vectors is that all expressions for evaluating the displacements are explicit functions of the original basis vectors. Calculation of any new basis vector $\mathbf{V}_{i}$ results in an additional term of the displacements expression (Eq. (20)) that is a function of the original vectors $\mathbf{r}_{j}(j=1,2, \ldots, i)$. Consequently, additional vectors can be considered without modifying the calculations that were carried out already.

The accuracy of the results achieved by the CA method have been demonstrated in previous studies [11-19]. The approximations provide accurate solutions even in cases where the series of the original basis vectors (the binomial series) diverges. Several criteria for evaluating the errors involved in the approximations have been developed [17,19]. These errors can be reduced when necessary by considering additional basis vectors.

The efficiency of reanalysis by the CA method, compared with complete analysis of the modified design, can be measured by various criteria, e.g. the CPU effort or the number of algebraic operations. It is then possible to relate the computational effort to various parameters such as the number of degrees of freedom, the number of basis vectors considered and the accuracy of the results. It has been shown that calculation of each basis vector involves about $2 \%$ of the CPU time needed for complete analysis. In many cases, a small number of basis vectors is sufficient to achieve adequate accuracy. For moderate changes in the design 2-3 vectors are often sufficient, while 5-6 vectors might be needed for large changes. Considering the latter number of basis vectors, results for various problems showed that the total CPU effort compared with complete analysis of the modified design can be reduced by more than $75 \%$.

\section{Exact Solutions for Low-rank Modifications}

Exact solutions are efficient in cases of low-rank modifications in the structure and are applicable to situations where the number of modified elements in the stiffness matrix is small. The rank of matrix $\Delta \mathbf{K}($ rank $\Delta \mathbf{K})$ is the dimension of the linear space spanned by its columns. Rank $\Delta \mathbf{K}$ is equal to the maximum number of linearly independent columns (or maximum number of linearly independent rows) of $\Delta \mathbf{K}$. Rank $\Delta \mathbf{K}$ is also equal to the order of the square sub-matrix of $\Delta \mathbf{K}$ of greatest order whose determinant does not vanish. Exact methods are usually based on the Sherman-Morrison [1] and Woodbury [2] formulae for the update of the inverse of a matrix. It has been shown recently [3] that various exact methods may be viewed as variants of these formulae.

In this section, exact solutions for low-rank modifications by the CA method are presented. In such cases, the basis vectors are selected in a different way, though similar to that of Eq. (8). Consider, for example, the case of simultaneous changes in $m$ truss members. The exact solution is achieved by the CA method if one basis vector is introduced for each changed member by [14]

$$
\mathbf{r}_{i}=\mathbf{K}_{0}^{-1} \Delta \mathbf{K}_{i} \mathbf{r}_{0} \quad i=1, \ldots, m
$$

where $\Delta \mathbf{K}_{i}$ is the contribution of the $i$ th member to $\Delta \mathbf{K}$. Note that the basis vectors in Eqs (8) and (21) are selected in a different way. If some of the basis vectors are linearly dependent, the exact solution is achieved for a smaller number of vectors. The exact solution for the case under consideration is given by

$$
\mathbf{r}=\mathbf{r}_{0}+\sum_{i=1}^{m} y_{i} \mathbf{r}_{i}
$$

This procedure is efficient in cases where the number of changed members is much smaller than the number of degrees of freedom. Exact solutions achieved by the CA method and the Sherman-MorrisonWoodbury formulae in such cases are equivalent.

The above procedure can be used to achieve exact solutions efficiently for geometrical changes, by viewing these changes as corresponding topological changes. For example, changing the coordinates of a single joint, it is possible to obtain the exact solution for the new design by viewing the change in the geometry as two successive changes in the topology. That is, all members connected to the joint are deleted, and new members are added at the modified location of the joint. Exact solutions 
for low-rank modifications in the geometry will be demonstrated by numerical examples in Section 6.1.

\section{Accurate Solutions for High-rank Modifications}

In general, the CA method does not provide exact solutions for high-rank modifications. In this section, two general cases where accurate solutions are achieved for high-rank changes in the geometry by the method are developed. It is shown that accurate solutions are achieved if the basis vectors come close to being linearly dependent. Such solutions are also achieved for nearly scaled geometries, when the angle between the two vectors representing the initial design and modified design is small. The solutions presented explain the high accuracy achieved by the CA method with only a small number of basis vectors.

\subsection{Nearly Scaled Geometries}

Scaling of the initial design is carried out by multiplying the initial stiffness matrix $\mathbf{K}_{0}$ by a positive scaling multiplier $\mu$ to obtain the modified matrix

$$
\mathbf{K}=\mu \mathbf{K}_{0}
$$

From Eqs (1), (4) and (23), it is clear that the exact displacements after scaling can be calculated directly by

$$
\mathbf{r}=\mu^{-1} \mathbf{r}_{0}
$$

The condition of Eq. (23) requires linear dependence of the stiffness matrix on the change in the design. In general, the elements of $\mathbf{K}$ are some nonlinear functions of the design variables. A typical case where the condition of Eq. (23) is satisfied is scaling of the geometry of a truss, where the lengths of all elements are multiplied by $\mu$ and their direction is unchanged.

Consider the above case where the modified design is a scaled design $\mu \mathbf{K}_{0}$, as given by Eq. (23). Then, from Eq. (5)

$$
\mathbf{B}=\mathbf{K}_{0}^{-1} \Delta \mathbf{K}=(\mu-1) \mathbf{I}
$$

where $\mathbf{I}$ is the identity matrix. It can be observed that the resulting basis vectors (Eq (8)) become linearly dependent

$$
\begin{aligned}
& \mathbf{r}_{1}=\mathbf{r}_{0} \\
& \mathbf{r}_{2}=-(\mu-1) \mathbf{r}_{0}
\end{aligned}
$$

$$
\mathbf{r}_{3}=(\mu-1)^{2} \mathbf{r}_{0}
$$

Thus, consideration of a single basis vector with a coefficient $y_{1}=\mu^{-1}$ will provide the exact solution as given by Eq. (24).

Consider the case where the change in the stiffness matrix $\Delta \mathbf{K}$ is expressed in terms of a corresponding change in the design variables $\mathbf{X}$ by

$$
\mathbf{X}=\mathbf{X}_{0}+\Delta \mathbf{X}
$$

The angle $\theta$ between the vector of the modified design $\mathbf{X}$ and the vector of initial design $\mathbf{X}_{0}$ is given by

$$
\cos \theta=\left(\mathbf{X}^{T} \mathbf{X}_{0}\right) /\left(|\mathbf{X}|\left|\mathbf{X}_{0}\right|\right)
$$

where $|\mathbf{X}|$ denotes the absolute value of $\mathbf{X}$. It will be shown in Section 6 that high accuracy is achieved with a small number of basis vectors for nearly scaled geometries, corresponding to small $\theta$ values. In general, more basis vectors are needed in cases of larger $\theta$ values. This result has been demonstrated in many examples where the space formed by the vectors $\mathbf{X}_{0}$ and $\mathbf{X}$ is considered. For the complete design space, smaller $\theta$ values do not always guarantee better approximations.

\subsection{Nearly Linear Dependent Basis Vectors}

It is shown in this section that when the reduced basis expression is equal to the exact solution, then the basis vectors are linearly dependent. Therefore, it would be reasonable to expect that accurate solutions will be achieved in cases were the basis vectors come close to being linearly dependent.

To obtain a convenient expression for the exact solution of the modified design, premultiply Eq. (4) by $\mathbf{K}_{0}^{-1}$ and substitute Eqs (1) and (5). Assuming for simplicity $\mathbf{R}=\mathbf{R}_{0}$, and premultipling the resulting equation by $(\mathbf{I}+\mathbf{B})^{-1}$ gives the exact modified displacements (see Eq. (6))

$$
\mathbf{r}=(\mathbf{I}+\mathbf{B})^{-1} \mathbf{r}_{0}
$$

To obtain a convenient expression for the approximate solution in terms of the assumed $s$ basis vectors, substitute the expressions of the basis vectors (Eq. (8)) into Eq. (16). The resulting CA expression is

$$
\mathbf{r}=y_{1} \mathbf{r}_{0}-y_{2} \mathbf{B} \mathbf{r}_{0}+y_{3} \mathbf{B}^{2} \mathbf{r}_{0}-\ldots+y_{s} \mathbf{B}^{s-1} \mathbf{r}_{0}
$$

Assuming that the approximate expression of Eq. (30) is equal to the exact solution of Eq. (29), premultiplying both equations by $(\mathbf{I}+\mathbf{B})$ and rearranging gives the linear expression 


$$
\mathbf{r}_{s+1}=\sum_{i=1}^{s} a_{i} \mathbf{r}_{i}
$$

where $a_{i}$ are scalar multipliers given by

$$
\begin{aligned}
a_{1} & =\left(y_{1}-1\right) / \mathrm{y}_{s} \\
a_{i} & =\left(y_{i}-y_{i-1}\right) / y_{s} \quad i=2,3, \ldots, s
\end{aligned}
$$

Equation (31) shows that when the reduced basis expression with $s$ terms is equal to the exact solution, then the $s+1$ basis vector is a linear combination of the previous $s$ vectors. That is, the $s+1$ basis vectors are linearly dependent. Consequently, it is expected that accurate solutions could be achieved in cases where the high-order basis vectors come close to being linearly dependent. Two basis vectors $\mathbf{r}_{i}$ and $\mathbf{r}_{i+1}$ are close to being linearly dependent if

$$
\cos \beta_{i+1}=\left(\mathbf{r}_{i}^{T} \mathbf{B} \mathbf{r}_{i}\right) /\left(\left|\mathbf{r}_{i}\right|\left|\mathbf{B} \mathbf{r}_{i}\right|\right) \cong 1
$$

where $\beta_{i+1}$ is the angle between the two vectors. It has been noted that the basis vectors determined by the CA method satisfy the condition of Eq. (33), as $i$ is increased, even in cases of very large changes in the geometry. In all the examples presented below linear dependence has been reached with only a small number of basis vectors. The degree of linear dependence of additional vectors could serve as a convergence criterion for the CA method, but better alternative criteria have been developed recently [19].

\section{Numerical Examples}

The solution approach presented in this study is suitable for different types of structure and design variables. For illustrative purposes, truss structures are considered in this section. Arbitrary units have been assumed and all cross-sectional areas equal to unity. The accuracy of the results is demonstrated only for displacements; similar accuracy has been achieved also for stresses.

\subsection{Low-rank Modifications (Nine-bar Truss)}

It has been noted that in cases of low-rank modifications, exact solutions can be achieved efficiently by viewing the change in the geometry as two successive changes in the topology. To demonstrate the solution process, assume the nine-bar truss with the initial geometry shown in Fig. 1(a). The object is to calculate displacements for the modified geometry shown in Fig. 1(b), where the coordinates of joint (a)

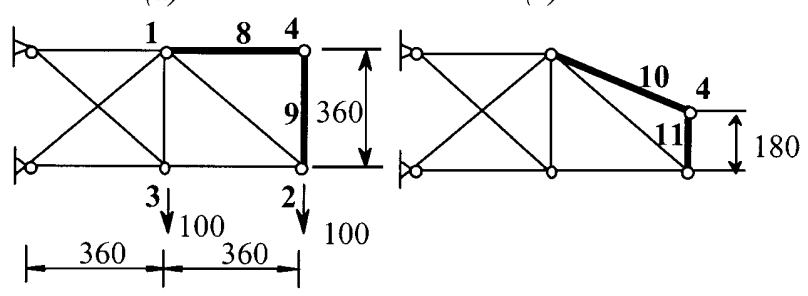

Fig. 1. (a) Initial geometry, nine-bar truss; (b) change in the geometry, viewed as deletion of members 8,9 and addition of members $10,11$.

4 have been changed. In this case, members 8,9 connected to joint 4 are first deleted and members 10,11 are then added in the new location of the joint.

The matrix of all changes in the stiffness is

$$
\Delta \mathbf{K}=\Delta \mathbf{K}(8,9)+\Delta \mathbf{K}(10,11)
$$

Since four members have been changed, four corresponding basis vectors are needed to achieve the exact solution. However, in the present case the matrices corresponding to the two vertical members 9, 11 are linearly dependent, therefore only three basis vectors are required. The resulting exact displacements achieved with three vectors are

$\mathbf{r}^{T}=$

$\{2.40,5.80,-3.60,15.19,-2.40,5.80,-2.30,15.19\}$

\subsection{Nearly Scaled Designs (Fifty-bar Truss)}

To illustrate nearly exact solution for structures with a larger number of degrees of freedom, consider the initial geometry of the cantilever truss shown in Fig. 2. The truss is subjected to a single load at the end, the modulus of elasticity is 10,000 , and the forty unknowns are the horizontal and the vertical displacements at joints 2 through 21, respectively. Two geometric variables have been considered, the
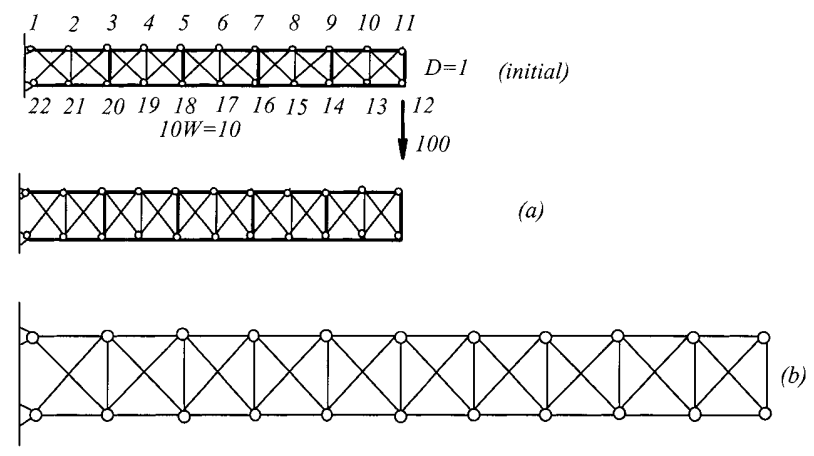

Fig. 2. Fifty-bar truss, initial and modified geometries. 
depth $D$ and the width $10 \mathrm{~W}$. Exact solution is achieved with a single basis vector for all designs where the ratio between the depth and the width of the truss is unchanged. The reason is that the vertical and the horizontal joint coordinates are changed simultaneously, such that the geometry is scaled (the lengths of all members are changed by the same percentage, whereas the direction cosines are unchanged).

Assuming the initial design $D=W=1.0$, two cases of geometrical changes have been solved:

(a) The changed geometry is given by $D=1.2$ (a change of $20 \%$ in the depth, Fig. 2(a)).

(b) The changed geometry is given by $D=2.0$, $W=1.9$ (a change of $100 \%$ in the depth and $90 \%$ in the width, Fig. 2(b).

Since the stiffness coefficients of many members have been changed, exact reanalysis is not efficient. Assuming only two basis vectors (first-order approximations, CA1), the results are given in Table 1. Comparing the results obtained for the two cases of geometrical modifications, it can be seen that better approximations have been achieved in case $b$, for larger changes in the geometry. The better results in case $b$ are attributed to the fact that the modified geometry is relatively close to a scaled geometry $(D=W)$, for which the CA1 provide the exact solution.

Table 1. First-order approximations of displacements, fifty-bar truss

\begin{tabular}{|c|c|c|c|c|c|}
\hline \multirow[t]{2}{*}{ Joint } & \multirow[t]{2}{*}{ Direction } & \multicolumn{2}{|c|}{ Case $(a)$} & \multicolumn{2}{|c|}{ Case $(b)$} \\
\hline & & CA1 & Exact & CA1 & Exact \\
\hline \multirow[t]{2}{*}{2} & $X$ & 0.09 & 0.08 & 0.20 & 0.20 \\
\hline & $\mathrm{Y}$ & 0.11 & 0.08 & 0.25 & 0.24 \\
\hline \multirow[t]{2}{*}{3} & $X$ & 0.16 & 0.15 & 0.38 & 0.38 \\
\hline & $\mathrm{Y}$ & 0.35 & 0.28 & 0.90 & 0.88 \\
\hline \multirow[t]{2}{*}{4} & $X$ & 0.22 & 0.21 & 0.54 & 0.54 \\
\hline & $\mathrm{Y}$ & 0.69 & 0.60 & 1.90 & 1.87 \\
\hline \multirow[t]{2}{*}{5} & $X$ & 0.26 & 0.27 & 0.68 & 0.67 \\
\hline & Y & 1.12 & 1.01 & 3.21 & 3.18 \\
\hline \multirow[t]{2}{*}{6} & $X$ & 0.29 & 0.31 & 0.79 & 0.79 \\
\hline & $\mathrm{Y}$ & 1.60 & 1.51 & 4.79 & 4.74 \\
\hline \multirow[t]{2}{*}{7} & $X$ & 0.32 & 0.35 & 0.88 & 0.88 \\
\hline & Y & 2.13 & 2.07 & 6.58 & 6.54 \\
\hline \multirow[t]{2}{*}{8} & $X$ & 0.34 & 0.38 & 0.95 & 0.96 \\
\hline & Y & 2.69 & 2.69 & 8.54 & 8.50 \\
\hline \multirow[t]{2}{*}{9} & $X$ & 0.35 & 0.40 & 1.01 & 1.01 \\
\hline & $\mathrm{Y}$ & 3.27 & 3.36 & 10.63 & 10.60 \\
\hline \multirow[t]{2}{*}{10} & $X$ & 0.35 & 0.41 & 1.04 & 1.04 \\
\hline & Y & 3.86 & 4.05 & 12.81 & 12.79 \\
\hline \multirow[t]{2}{*}{11} & $X$ & 0.35 & 0.42 & 1.05 & 1.05 \\
\hline & $\mathrm{Y}$ & 4.45 & 4.75 & 15.02 & 15.02 \\
\hline
\end{tabular}

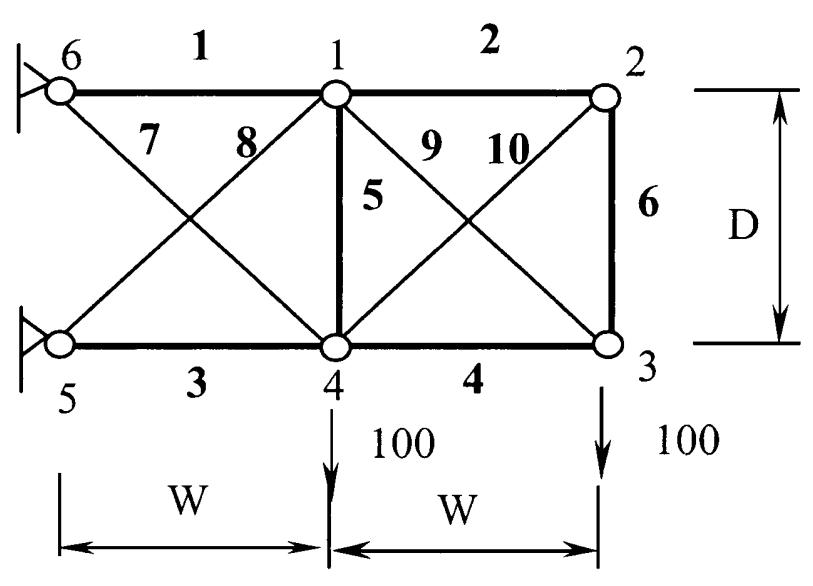

Fig. 3. Ten-bar truss.

\subsection{Nearly Linear Dependent Basis Vectors (Ten-bar Truss)}

Consider the ten-bar truss shown in Fig. 3 with two geometrical variables, the depth $D$ and the width $2 W$. Assuming the initial design $W=D=360$, then for $W=D=720$ (increase of $100 \%$ in the length of all members), the exact solution is simply

$\mathbf{r}^{T}=2 \mathbf{r}_{0}^{T}=$

$\{4.68,11.16,5.64,25.30,-6.34,26.26,-4.92,12.02\}$

To demonstrate the accuracy of low-order approximations achieved by the CA method for various geometrical modifications, assume first-order (CA1) and second-order (CA2) approximations of displacements for the following three cases shown in Fig. 4:

(a) $W=360, D=540$ (increase of $50 \%$ in the depth).

(b) $W=360, D=720$ (increase of $100 \%$ in the depth).

(c) $W=180, D=720$ (increase of $100 \%$ in the depth and decrease of $50 \%$ in the length).

From the results summarised in Table 2, it can be observed that the changes in geometry result in significant changes in the displacements. The high accuracy of the results is explained by the $\beta$ values (Eq. 34)) obtained for the basis vectors. In case (b),
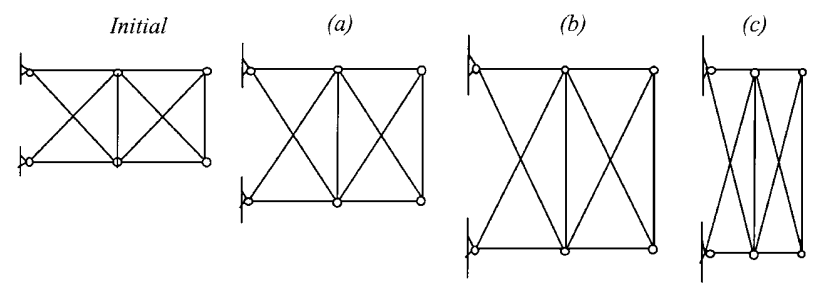

Fig. 4. Ten-bar truss, initial and modified geometries. 
Table 2. First- and second-order approximations, ten-bar truss

\begin{tabular}{|c|c|c|c|c|c|c|c|c|c|c|}
\hline \multirow[t]{2}{*}{$L$} & \multirow[t]{2}{*}{$D$} & \multirow[t]{2}{*}{ Method } & \multicolumn{8}{|c|}{ Displacements } \\
\hline & & & 1 & 2 & 3 & 4 & 5 & 6 & 7 & 8 \\
\hline 360 & 360 & Exact & 2.34 & 5.58 & 2.82 & 12.65 & -3.17 & 13.13 & -2.46 & 6.01 \\
\hline 360 & 540 & $\begin{array}{l}\text { CA1 } \\
\text { CA2 } \\
\text { Exact }\end{array}$ & $\begin{array}{l}1.49 \\
1.53 \\
1.55\end{array}$ & $\begin{array}{l}4.02 \\
3.93 \\
3.94\end{array}$ & $\begin{array}{l}1.71 \\
1.81 \\
1.82\end{array}$ & $\begin{array}{l}7.86 \\
7.83 \\
7.84\end{array}$ & $\begin{array}{l}-2.06 \\
-2.17 \\
-2.18\end{array}$ & $\begin{array}{l}8.40 \\
8.46 \\
8.47\end{array}$ & $\begin{array}{l}-1.60 \\
-1.64 \\
-1.66\end{array}$ & $\begin{array}{l}4.48 \\
4.45 \\
4.44\end{array}$ \\
\hline 360 & 720 & $\begin{array}{l}\text { CA1 } \\
\text { CA2 } \\
\text { Exact }\end{array}$ & $\begin{array}{l}1.17 \\
1.14 \\
1.15\end{array}$ & $\begin{array}{l}3.78 \\
3.67 \\
3.67\end{array}$ & $\begin{array}{l}1.26 \\
1.34 \\
1.34\end{array}$ & $\begin{array}{l}6.72 \\
6.62 \\
6.60\end{array}$ & $\begin{array}{l}-1.61 \\
-1.68 \\
-1.66\end{array}$ & $\begin{array}{l}7.29 \\
7.35 \\
7.36\end{array}$ & $\begin{array}{l}-1.28 \\
-1.24 \\
-1.25\end{array}$ & $\begin{array}{l}4.27 \\
4.25 \\
4.24\end{array}$ \\
\hline 180 & 720 & $\begin{array}{l}\text { CA1 } \\
\text { CA2 } \\
\text { Exact }\end{array}$ & $\begin{array}{l}0.43 \\
0.31 \\
0.29\end{array}$ & $\begin{array}{l}2.59 \\
2.52 \\
2.47\end{array}$ & $\begin{array}{l}0.36 \\
0.43 \\
0.33\end{array}$ & $\begin{array}{l}3.83 \\
3.86 \\
3.85\end{array}$ & $\begin{array}{l}-0.59 \\
-0.55 \\
-0.42\end{array}$ & $\begin{array}{l}4.27 \\
4.44 \\
4.53\end{array}$ & $\begin{array}{l}-0.50 \\
-0.35 \\
-0.31\end{array}$ & $\begin{array}{l}2.97 \\
2.98 \\
2.94\end{array}$ \\
\hline
\end{tabular}

for example, the first two values are cos $\beta_{1,2}=0.9912, \cos \beta_{2,3}=0.9999$. These results show that the basis vectors determined by the CA method are close to being linearly dependent.

\subsection{High-rank Modifications (Hundred and Thirty-bar Truss)}

To illustrate high-rank modifications in the geometry of larger structures, consider the hundred and thirtybar truss shown in Fig. 5. The ten-story three-bay truss is subjected to ten equal horizontal loads of 10.0. Two cases of changes in the geometry have been considered:

\section{Case a}

Change in the location of the 3rd column from the left (Fig. 6(a)). The following changes have been considered (for the initial geometry $X=200$ ):

(a1) $\mathrm{X}=250$ (increase of $25 \%$ ).

(a2) $X=300$ (increase of $50 \%$ ).

(a3) $X=350$ (increase of $75 \%$ ).

Case b

Symmetrical changes in the location of the outer joints (Fig. 6(b)). The following changes have been considered (for the initial geometry $X=200$ ):

(b1) $\mathrm{X}=100$ (decrease of $50 \%$ ).

(b2) $X=50$ (decrease of $75 \%$ ).

The maximum horizontal displacements at the top left joint obtained for various numbers of basis vectors are summarised in Table 3, and the corresponding errors in the approximations are shown in Table 4. It can be seen that the larger the change in the geometry, the more basis vectors are needed

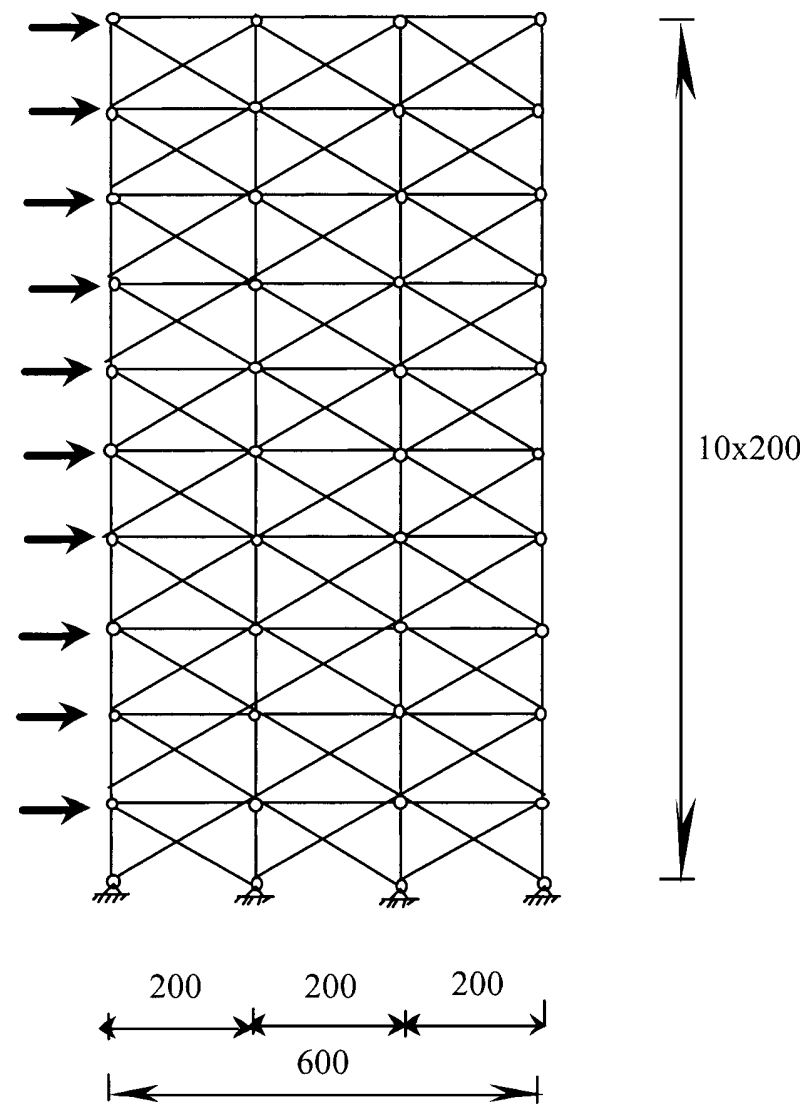

Fig. 5. Ten-story three-bay truss, initial geometry.

to achieve accurate results. Similar errors have been obtained for other displacements.

\section{Conclusions}

A reanalysis method intended for highly nonlinear geometrical changes in structures has been 


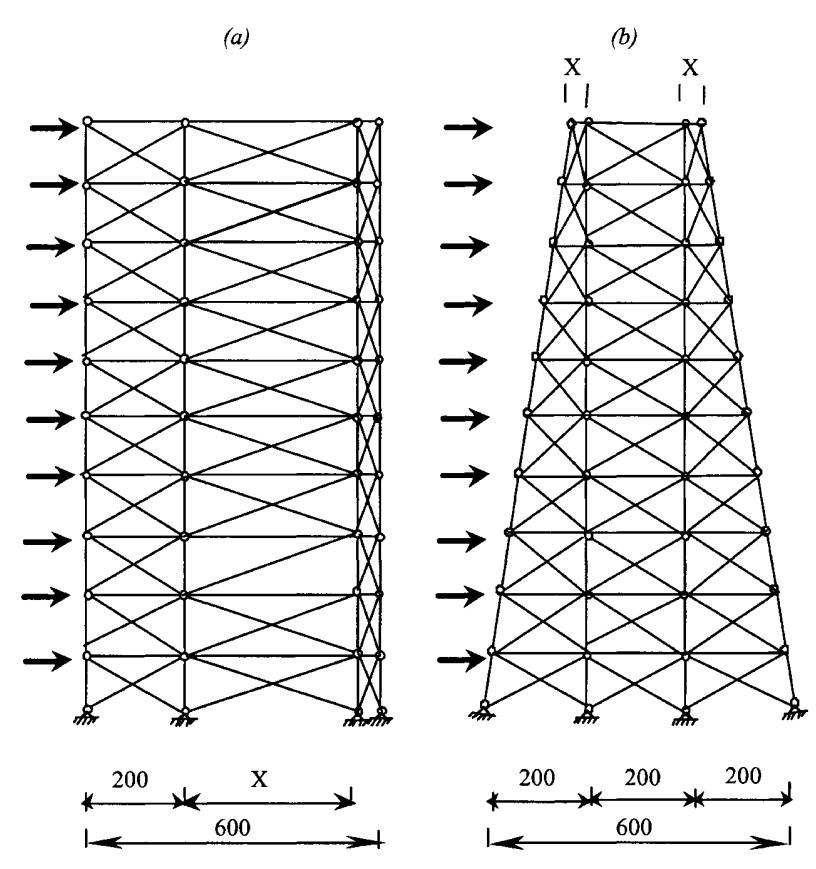

Fig. 6. Ten-story truss, modified geometries.

Table 3. Maximum horizontal displacements, hundred and thirty-bar truss

\begin{tabular}{|c|c|c|c|c|c|c|}
\hline \multirow{2}{*}{\multicolumn{2}{|c|}{$\begin{array}{l}\text { Case } \\
\text { Geometry }\end{array}$}} & \multicolumn{3}{|c|}{ (a) } & \multicolumn{2}{|c|}{ (b) } \\
\hline & & (a1) & (a2) & (a3) & (b1) & (b2) \\
\hline & 2 & 14.06 & 13.12 & 11.42 & 13.37 & 10.67 \\
\hline Number of & 3 & 14.26 & 13.61 & 12.68 & 15.28 & 13.22 \\
\hline Basis & 4 & & 13.82 & 13.33 & 15.61 & 15.31 \\
\hline \multirow[t]{2}{*}{ vectors } & 5 & & & 13.52 & & 15.99 \\
\hline & 6 & & & & & 16.18 \\
\hline Exact & & 14.26 & 13.83 & 13.56 & 15.63 & 16.28 \\
\hline
\end{tabular}

Table 4. Errors (\%) in maximum horizontal displacements, hundred and thirty-bar truss

\begin{tabular}{|c|c|c|c|c|c|c|}
\hline \multirow{2}{*}{$\begin{array}{l}\text { Case } \\
\text { Geometry }\end{array}$} & & \multicolumn{3}{|c|}{ (a) } & \multicolumn{2}{|c|}{ (b) } \\
\hline & & (a1) & (a2) & (a3) & (b1) & (b2) \\
\hline & 2 & 1.4 & 5.1 & 15.8 & 14.4 & 34.5 \\
\hline Number of & 3 & 0 & 1.6 & 6.5 & 2.2 & 18.8 \\
\hline Basis & 4 & & 0.1 & 1.7 & 0.1 & 16.0 \\
\hline vectors & 5 & & & 0.3 & & 1.8 \\
\hline & 6 & & & & & 0.7 \\
\hline
\end{tabular}

presented. Using the solution procedure, accurate approximations of displacements are obtained efficiently for large changes in the geometry. Most of the computational effort is needed for calculation of the basis vectors. This calculation involves only forward and backward substitutions in cases where the initial stiffness matrix is given in a decomposed form from initial analysis of the structure. It has been found that calculation of each basis vector involves about $2 \%$ of the CPU time needed for complete analysis. In many cases, a small number of basis vectors is sufficient to achieve adequate accuracy. For moderate changes in the design, 2-3 vectors are often sufficient, while 5-6 vectors might be needed for large changes. Considering the latter number of basis vectors, results for various problems showed that the total CPU effort, compared with complete analysis of the modified design, has been reduced by more than $75 \%$.

Exact solutions are obtained efficiently for changes in a small number of elements (low-rank modifications in the structure). Such solutions are also obtained in cases of scaling of the initial geometry, or when a basis vector is a linear combination of the previous vectors. Accurate solutions are achieved for nearly scaled geometries, when the angle between the two vectors representing the initial design and modified design is small, or when the basis vectors come close to being linearly dependent. The exact solutions presented in the paper explain the high accuracy achieved by the approximations with only a small number of basis vectors.

In the examples demonstrated, accurate approximations have been achieved for some discrete structures. The accuracy of the results illustrates the potential of the CA method in cases of large changes in the geometry. The procedure presented might prove useful for various problems of geometrical changes, such as shape optimisation of continuum structures.

\section{Acknowledgements}

This work has been partially supported by the Automotive Research Center under the sponsorship of the US Army TACOM, and by the General Motors Satellite Research Laboratory at the University of Michigan, Ann Arbor. The support is gratefully acknowledged. The authors wish to thank Matt Parkinson, graduate student, for his help in solving the numerical examples. 


\section{References}

1. Sherman, J., Morrison, W. J. (1949) Adjustment of an inverse matrix corresponding to changes in the elements of a given column or a given row of the original matrix. Ann Math, Statist, 20, 621

2. Woodbury, M. (1950) Inverting modified matrices. Memorandum Report 42, Statistical research group, Princeton University, Princeton, NJ

3. Akgun, M. A., Garcclon, J. H., Haftka, R. T. (1998) Fast exact linear and nonlinear structural reanalysis and the Sherman-Morrison-Woodbury formulas. NASA/ISSMO First Internet Conference on Approximations and Fast Reanalysis in Engineering Optimization

4. Kirsch, U. (1981) Optimum Structural Design. New York, McGraw Hill

5. Barthelemy, J.-F. M., Haftka, R. T. (1993) Approximation concepts for optimum structural design - a review. Structural Optimization, 5, 129-144

6. Kirsch, U. (1993) Structural Optimizations, Fundamentals and Applications. Heidreorg, Springer-Verlag

7. Noor, A. K. (1994) Recent advances and applications of reduction methods. Appl Mech Rev, 47, 125-146

8. Sobieszezanski-Sobieski, J., Haftka, R. T. (1997) Multidisciplinary aerospace design optimization: survey and recent developments. Structural Optimization, 14, $1-23$

9. Schmit, L. A., Farshi, B. (1974) Some approximation concepts for structural synthesis. AIAA J, 11, 489-494

10. Svanberg, K. (1987) The method of moving asymptotes - a new method for structural optimization. Int J Num Meth Engrg, 24, 359-373

11. Kirsch, U. (1993) Efficient reanalysis for topological optimization. Structural Optimization, 6, 143-150

12. Kirsch, U. (1999) Efficient-accurate approximations for structural optimization. AIAA Journal, 37, 16631669

13. Kirsch, U. (2000) Combined approximations - a general approach for structural optimization. Structural Optimization, 20, 97-106

14. Kirsch, U., Liu S. (1995) Exact structural reanalysis by a first-order reduced basis approach. Structural Optimization, 10, 153-158

15. Kirsch, U., Liu, S. (1997) Structural reanalysis for general layout modifications. AIAA Journal, 35, 382-388

16. Kirsch, U., Papalambros P. Y. (2001) Exact and accurate solutions in the approximate reanalysis of structures. AIAA Journal

17. Kirsch, U., Papalambros P. Y. (2001) Structural reanalysis for topological modifications - a unified approach. Structural Optimization

18. Leu, L.-J., Huang, C.-W. (1998) A reduced basis method for geometric nonlinear analysis of structures. IASS Journal, 39, 71-75

19. Kirsch, U., Kocvara, M., Zowe, J. Accurate reanalysis of structures by a preconditioned conjugate gradient method. Submitted 